\title{
Optimization-Based Market-Clearing Procedure with EVs Aggregator Participation
}

\author{
Miguel A. López, José A. Aguado, Sebastián de la Torre and Miriam Figueroa \\ Electrical Engineering Department, Universidad de Málaga (UMA), Spain \\ Email:malopezperez@uma.es, storre@uma.es, jaguado@uma.es, mfigueroa@uma.es
}

\begin{abstract}
For the upcoming new generation of electric power systems, i.e. smart grids, one of the most important challenges is to achieve an adequate economic and technical management involving the different agents in the process.

In order to deliver the available power from suppliers to consumers, a market-clearing mechanism is needed. At the same time, technical operation calls for controlling that technical limits are not reached to preserve the security of the system. In this environment, Electric Vehicles (EVs) are gaining importance both in economic and technical issues.

In this paper, an optimization-based approach is proposed for clearing the market in a smart grid. The traditional participants in energy markets are included in the formulation, stressing the role of EVs aggregators. The results presented in this paper illustrate the influence of EVs in the market-clearing procedure. The benefits for the system and EVs aggregators are also studied.
\end{abstract}

Index Terms-EVs aggregator, market-clearing, optimization

\section{INTRODUCTION}

In order to facilitate the integration of EVs under the concept of smart grid it is interesting the development of specific tools that allow them to participate in the market complying with the necessary security restrictions in the system at the same time. EVs' incorporation in energy markets cannot be done individually because the amount of energy they can offer is very small compared to other agents' offers. Therefore, their participation makes sense under an EVs aggregator entity that can merge the individual requirements and constraints of EVs to submit bids to the market [1], [2].

With respect to the inclusion of an aggregator in a market environment several approaches have been developed. An algorithm to forecast EV demand and prices, is used to determine optimal scheduling in [3]. Two different approaches for allowing an aggregator agent to participate in day-ahead markets are presented in [4] and [5], showing advantages and drawbacks of both and supporting the optimization formulation with a complete numerical analysis. Charging and discharging of EVs are optimized in [6] where an aggregator agent allows an EV fleet to participate in the market. Specific algorithms are used to avoid technical problems arising from EVs charging in [7]. The calculation of EV charging to avoid congestion in grid lines is studied in [8]. The effects of two scheduling models for EV charging on the day-ahead market is analysed in [9]. In [10], authors propose a model where an EVs aggregator can coordinate EVs charging and it can also offer services like Vehicle-to-Grid (V2G), energy and reserve. Finally, EV regulation services are investigated under three different strategies in [11]. All these works and other present in the literature show the great importance of the aggregator role for allowing EVs to participate actively in the electricity market.

In this paper, a market-clearing model based on optimization, including the role of EVs aggregator, is proposed. The objective function aims at maximizing the difference between the price the consumers are willing to pay for their energy and the price the suppliers offer for their production; clearing the market and satisfying the security requirements at the same time. Along with the traditional agents involved in a market-clearing procedure, such as suppliers and consumers, the effect, constraints and role of EVs' aggregators are studied and described. The main contribution of this paper is the inclusion in the model of EVs' aggregators that can bid for buying or selling energy satisfying the mobility requirements of the fleet they represent completing the formulation shown in [12].

\section{OPTIMIZATION-BASED APPROACH MODEL}

To define the optimization problem it is necessary to take into account the contribution of the different participants in the process.

This problem is formulated as the maximization of the sum of four terms:

$$
\begin{aligned}
\text { maximize } & z_{S}+z_{C}+z_{B}+z_{A} \\
\text { s.t. } & \mathbf{f}_{S} \leq \mathbf{0}, \quad \mathbf{f}_{C} \leq \mathbf{0}, \quad \mathbf{f}_{B} \leq \mathbf{0} \\
& \mathbf{f}_{A} \leq \mathbf{0}, \quad \mathbf{g}=\mathbf{0}
\end{aligned}
$$

where $z_{S}, z_{C}, z_{B}$ and $z_{A}$ are the functions that define the utility for suppliers, consumers, bilateral contracts and EVs aggregators respectively and $\mathbf{f}_{S}, \mathbf{f}_{C}, \mathbf{f}_{B}$ and $\mathbf{f}_{A}$ represent the corresponding inequality constraints. Function $\mathbf{g}$ includes the equations for balancing supply and demand in the grid.

For the market to take place, as shown above, four elements have been considered:

- $S$, set of suppliers that submit offers for selling power and spinning-reserve, where $S=1,2, \ldots, n_{s}$.

- $C$, set of consumers that bid for buying power, where $C=1,2, \ldots, n_{c}$.

- $B$, set of bilateral agreements, where $B=1,2, \ldots, n_{b}$.

- $A$, set of EVs aggregators, where $A=1,2, \ldots, n_{a}$.

The corresponding contribution to the objective function and constraints for each participant are given next. 


\section{A. Suppliers}

To participate in the market, suppliers have to submit the power available and the power dedicated to spinning reserve, along with their offer prices, in each time period, which typically are given in a day-ahead basis. This way, the suppliers function $z_{S}$ in (1) can be written as:

$$
z_{S}=-\sum_{t=1}^{n_{t}} \sum_{i=1}^{n_{s}}\left(\mu_{t}^{i} \cdot p_{t}^{i}+\nu_{t}^{i} \cdot r_{t}^{i}\right)
$$

where $p_{t}^{i}$ and $r_{t}^{i}$ are the hourly amounts of power and spinning -reserve put at stake and $\mu_{t}^{i}$ and $\nu_{t}^{i}$ are the corresponding hourly offer prices, respectively, for each supplier $i$ and time period $t$ in monetary units (m.u.) per MWh. The parameter $n_{t}$ defines the time horizon.

Conventional generators have to conform with the following equations regarding operation:

$$
\begin{gathered}
0 \leq p_{t}^{i}+r_{t}^{i} \leq p_{\text {max }}^{i} \quad \forall i \in S, \forall t \in T \\
p_{t+1}^{i}-p_{t}^{i} \leq \triangle \bar{u}_{i} \quad \forall i \in S, \forall t \in T \\
p_{t}^{i}-p_{t+1}^{i} \leq \nabla \bar{u}_{i} \quad \forall i \in S, \forall t \in T
\end{gathered}
$$

Equation (3) expresses that power output for generators cannot be higher than the upper technical limit $p_{\text {max }}^{i}$. Equations (4) and (5) model ramping rates limits.

The nodal power injection $P_{t, n}^{S}$ for the suppliers is:

$$
P_{t, n}^{S}=\sum_{i \propto n} p_{t}^{i} \quad \forall t \in T, \forall n \in N
$$

where $i \propto n$ defines all suppliers $i$ connected to a bus $n$.

\section{B. Consumers}

Consumers participating in the market bid for demand in the time horizon for buying the energy they need. The consumers function $z_{C}$ in (1) is:

$$
z_{C}=\sum_{t=1}^{n_{t}} \sum_{j=1}^{n_{c}} \lambda_{t}^{j} \cdot \underline{d}_{t}^{j}
$$

where $\underline{d}_{t}^{j}$ is the hourly amount of dispatchable demand and $\lambda_{t}^{j}$ and is the corresponding hourly offer price, respectively, for each consumer $j$ and time period $t$.

The maximum hourly dispatchable demand is limited to a fraction $\tau_{t}^{j}$ of the total demand $d_{t}^{j}$ :

$$
0 \leq \underline{d}_{t}^{j} \leq \tau_{t}^{j} \cdot d_{t}^{j} \quad \forall j \in C, \forall t \in T
$$

The nodal power demand $D_{t, n}^{C}$ for the consumers at bus $n$ is the sum of non-dispatchable and dispatchable demand:

$$
D_{t, n}^{C}=\sum_{j \propto n}\left(\left(1-\tau_{t}^{j}\right) \cdot d_{t}^{j}+\underline{d}_{t}^{j}\right) \quad \forall t \in T, \forall n \in N
$$

Consumers bid for only the dispatchable demand, meanwhile the remainder, the inelastic demand, is included in the power balance regardless of the price.

\section{Bilateral agreements}

For bilateral agreements it is necessary to establish the schedule for the hourly amounts that are under the contract between specific sellers and buyers:

$$
z_{B}=\sum_{t=1}^{n_{t}} \sum_{b=1}^{n_{b}}\left(\varepsilon_{t}^{b} \cdot \nabla c_{t}^{b}-\vartheta_{t}^{b} \cdot \triangle c_{t}^{b}\right)
$$

where the pairs $\left(\varepsilon_{t}^{b}, \nabla c_{t}^{b}\right),\left(\vartheta_{t}^{b}, \triangle c_{t}^{b}\right)$ define the corresponding couple of schedule decremental and incremental, price and energy, respectively.

The amount of energy under contract is represented by $E_{t}^{b}$; the decremental and incremental constraints are given by:

$$
\begin{aligned}
& 0 \leq \triangle c_{t}^{b} \leq \bar{\varphi}_{b} \cdot E_{t}^{b} \\
& 0 \leq \nabla c_{t}^{b} \leq \underline{\varphi}_{b} \cdot E_{t}^{b}
\end{aligned}
$$

where $\bar{\varphi}_{b}$ and $\underline{\varphi}_{b}$ are the fractions of the contracted energy that the supplier is willing to sell or buy in the market.

The nodal contribution from bilateral contracts is:

$$
P_{t, n}^{B}=\sum_{b \propto n}\left(E_{t}^{b}+\nabla c_{t}^{b}-\triangle c_{t}^{b}\right)-\sum_{\bar{b} \propto n} E_{t}^{\bar{b}} \forall t \in T, \forall n \in N
$$

where $b \propto n$ and $\bar{b} \propto n$ define the seller and buyer connection buses to the grid and $E_{t}^{b}=E_{t}^{\bar{b}}$.

\section{EVs aggregator model}

Under this model EVs can act as consumers, bidding for demand, or instead they can act as suppliers, submitting offers and, therefore, performing V2G; bidding through an EVs aggregator agent. It is assumed that EVs are bundled in groups with similar movement patterns. The EVs aggregator is responsible for satisfying the mobility requirements of the group; it also manages V2G performance whenever necessary. The hourly bids give the aggregator the possibility to allocate the charging or discharging when it is more economically beneficial. The aggregator can coordinate one or several groups of EVs located in different buses of the grid.

The EV contribution is determined through the EV pattern, that is, the time periods when EVs are available in a particular node for charging or discharging, and the time periods when they are travelling. Assuming that a group of EVs cannot sell and buy energy in the same time period, the clearing market procedure will give, on the one hand, the allocation of the EV charging that meet the requirements regarding mobility and, on the other hand, the EV discharging.

The aggregator function $z_{A}$ is expressed in the following way:

$$
z_{A}=\sum_{t=1}^{n_{t}} \sum_{a=1}^{n_{a}}\left(\beta_{t}^{a} \cdot p_{t}^{c, a}-\alpha_{t}^{a} \cdot p_{t}^{d, a}\right)
$$

where $p_{t}^{d, a}$ and $p_{t}^{c, a}$ are the discharging power offered and the charging power required for EVs belonging to an aggregator $a$, respectively; and $\alpha_{t}^{a}$ and $\beta_{t}^{a}$ are the corresponding offer prices. 
Note that variables $p_{t}^{c, a}$ and $p_{t}^{d, a}$ represent the total hourly charging and discharging power for a group $g$ of $n_{a}^{g} \mathrm{EVs}$, belonging to an aggregator $a$, with identical movement pattern. Parameters $\beta_{t}^{a}$ and $\alpha_{t}^{a}$ can depend on the bus where EVs are connected and are also the instruments to control the charging and discharging. As it will be stated later, a group of EVs will behave in the same way, that is, they all will charge or discharge at the same time. EVs in the same node can be also included in different groups.

The constraints considered regarding the maximum charging and discharging power are:

$$
\begin{aligned}
& 0 \leq p_{t}^{c, a} \leq P_{n}^{\max } \cdot n_{a}^{g} \quad \forall a \in A, \forall t \in T, \forall n \in N \\
& 0 \leq p_{t}^{d, a} \leq P_{n}^{\max } \cdot n_{a}^{g} \quad \forall a \in A, \forall t \in T, \forall n \in N
\end{aligned}
$$

where $P_{n}^{\max }$ is the nodal rate power.

The nodal contribution of EVs is expressed as:

$$
P_{t, n}^{A}=\sum_{a \propto n}\left(p_{t}^{d, a}-p_{t}^{c, a}\right) \quad \forall t \in T, \forall n \in N
$$

The battery State of Charge (SOC) $S_{t}^{a}$ for one EV in a group $g$ has to comply with the following constraints:

$$
\begin{gathered}
S_{t+1}^{a}=S_{t}^{a}+\eta_{c} \cdot \frac{p_{t}^{c, a}}{n_{a}^{g}}-\left(1 / \eta_{d}\right) \cdot \frac{p_{t}^{d, a}}{n_{a}^{g}}-C_{t}^{a} \\
\forall a \in A, \forall t \in T \\
S_{\text {min }}^{a} \leq S_{t}^{a} \leq S_{\text {max }}^{a} \quad \forall a \in A, \forall t \in T \\
S_{t}^{a}=S_{\text {max }}^{a} \text { if } t=t^{*} \quad \forall a \in A \\
S_{t_{i}}^{a} \leq S_{t_{f}}^{a} \quad \forall a \in A
\end{gathered}
$$

Equation (18) guarantees the transition of the SOC $S_{t}^{a}$ according to the EV charging, EV discharging and consumption due to mobility $C_{t}^{a}$ in the current time period through the corresponding charging, and discharging efficiencies, $\eta_{C}, \eta_{D}$. Note that $p_{t}^{c, a} / n_{a}^{g}$ and $p_{t}^{d, a} / n_{a}^{g}$ are the absorbed and drawn power for a single EV respectively. Equation (19) limits the maximum and minimum value for the SOC in $S_{\max }^{a}$ and $S_{\text {min }}^{a}$. Finally, (20) forces the maximum value for the SOC in time period $t^{*}$, early morning, and (21) establishes that the final SOC has to be higher or equal to the initial SOC.

\section{E. Grid model}

Regarding security constrains a DC power flow model has been adopted:

$$
P_{m, n}^{t}=B_{m, n} \cdot\left(\theta_{t}^{m}-\theta_{t}^{n}\right) \quad \forall m, n \in N, \forall t \in T, m \neq n
$$

where $P_{m, n}^{t}$ is the active power flow in the line $m-n, B_{m, n}$ is the line susceptance and $\theta_{m}, \theta_{n}$ are the phase angles.

The limits for active power flow are defined by:

$$
-P_{m, n}^{\max } \leq P_{m, n}^{t} \leq P_{m, n}^{\max } \forall m, n \in N, \forall t \in T, m \neq n
$$

The power balance in every bus of the grid is guaranteed with:

$$
\begin{aligned}
& P_{t, n}^{S}+P_{t, n}^{B}+P_{t, n}^{A}+\sum_{m \prec n} P_{m, n}^{t}=D_{t, n}^{C}+\sum_{m \prec n} P_{m, n}^{L, t} \\
& \forall n \in N, \forall t \in T, m \neq n
\end{aligned}
$$

where $m \prec n$ defines all the $m$ buses connected to $n$. Note that $P_{m, n}^{L, t}$ allocates $50 \%$ of the losses in line $m-n$ to node $n$ and it is computed through a quadratic model:

$$
P_{m, n}^{L, t}=B_{m, n} \cdot\left(\theta_{t}^{m}-\theta_{t}^{n}\right)^{2} \quad \forall m, n \in N, \forall t \in T, m \neq n
$$

\section{CASE STUDY}

The case study network is based on the data and characteristics for the IEEE-RTS 24-bus system [13], composed of 38 lines, 10 generating units, 14 consumption units, 2 bilateral contracts and 15 buses with EVs, Fig. 1.

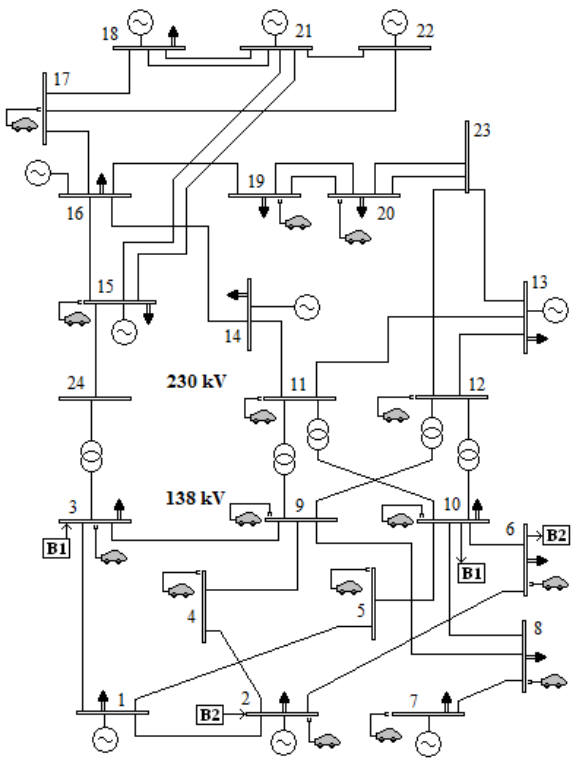

Fig. 1. 24-bus system

The technical data for each generator, namely the maximum power output and ramps rates, are given in Table I.

TABLE I

TECHNICAL DATA FOR GENERATING UNITS

\begin{tabular}{c|ccc} 
Bus & $p_{\max }(\mathrm{MW})$ & $\triangle \bar{u}(\mathrm{MW})$ & $\nabla \bar{u}(\mathrm{MW})$ \\
\hline \hline 1 & 100 & 50 & 50 \\
\hline 2 & 100 & 50 & 50 \\
\hline 7 & 76 & 40 & 40 \\
\hline 13 & 76 & 40 & 40 \\
\hline 14 & 20 & 5 & 5 \\
\hline 15 & 76 & 40 & 40 \\
\hline 16 & 76 & 40 & 40 \\
\hline 18 & 20 & 10 & 10 \\
\hline 21 & 20 & 20 & 20 \\
\hline 22 & 20 & 7 & 7 \\
\hline
\end{tabular}

The model is solved for a 24 hour horizon. The load for three representative consumers, located in buses 6,14 and 18, is given in Fig. 2. For the rest of the consumers the trend is similar with significant loads towards the end of the day. The amount of dispatchable demand depends on the time period but it is around $12 \%$ for all the consumers. 


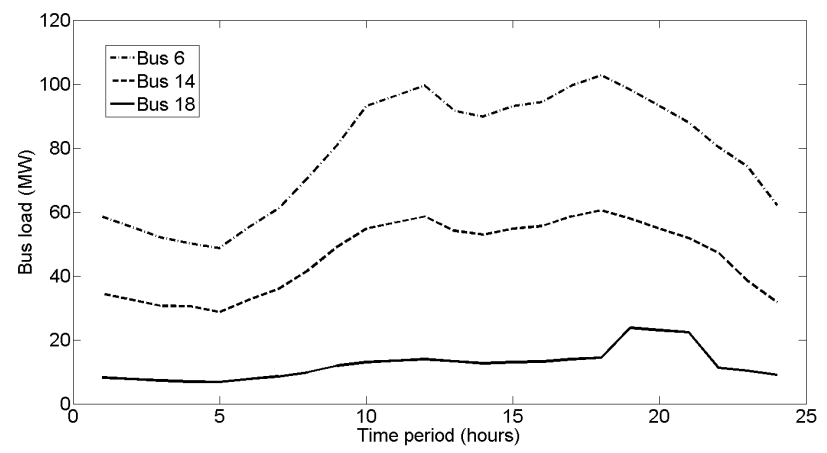

Fig. 2. Hourly consumers load

Regarding the bilateral contracts, the energy selling and purchase bids are 8,9 and 4,3 , in m.u./MWh, respectively for both. The amount of energy the supplier is willing to sell or buy in the market is assumed to be $20 \%$ for both contracts. The volume of energy contracted is different for every time period and it is between $10 \mathrm{MW}$ and $30 \mathrm{MW}$.

Finally, 7800 EVs have been considered in the system, this roughly constitutes $5 \%$ of the system power capacity. The EVs have been distributed into 30 groups of $260 \mathrm{EVs}$ each and with 2 groups in each bus. It is assumed that all the EVs have the same characteristics, the maximum and minimum battery level are $16.5 \mathrm{kWh}$ and $1.65 \mathrm{kWh}$ respectively. A value of $3.7 \mathrm{~kW}$ has been chosen for the maximum EV charging and discharging meanwhile, the corresponding efficiencies, $\eta_{C}$ and $\eta_{D}$, are 0.95 and 0.98 .

It is considered that each EV performs two journeys a day. The outward journey takes place in the early morning and the return journey in the afternoon, or evening. Fig. 3 gives the hourly configuration of the journeys and the total consumption in MWh for all the EVs. It is also assumed that EVs are attached to a particular bus in the system, that is, when EVs move, connection points at the origin and destination are linked to the same transmission grid bus. Note that the battery consumption represented in Fig. 3 takes place when EVs are moving; this is not a load for the grid.

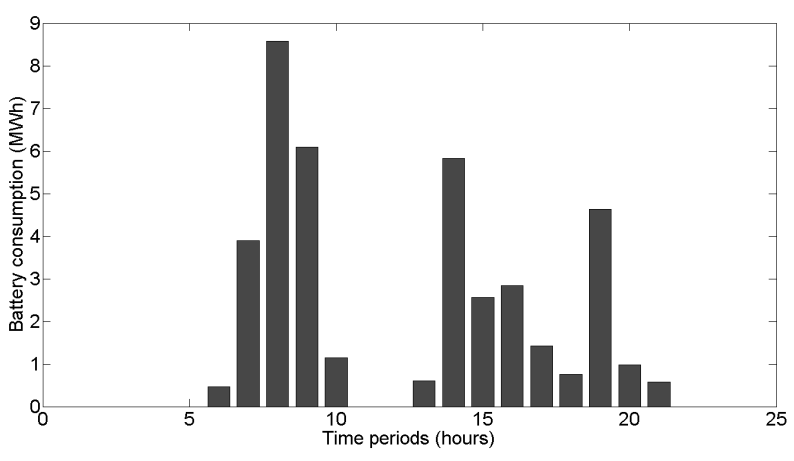

Fig. 3. EV battery consumption in trips

The hourly offer prices for suppliers were adapted from generation costs given in [13] depending on the different production technologies. The bidding prices for dispatchable demand are calibrated to provide illustrative results. Finally, EVs offer prices were chosen to represent realistic situations.

\section{RESULTS}

In this section, the most relevant results of applying the proposed model to the described system are presented.

One of the most important results of the clearing market model is the nodal price. In practice, nodal prices determine, for example, the dispatch of generators and demand. In this work, it is assumed that the system capacity is high enough so no congestion in lines takes place. Thus, nodal prices, although different because of losses, are very close. Fig 4 shows the price for bus 1 .

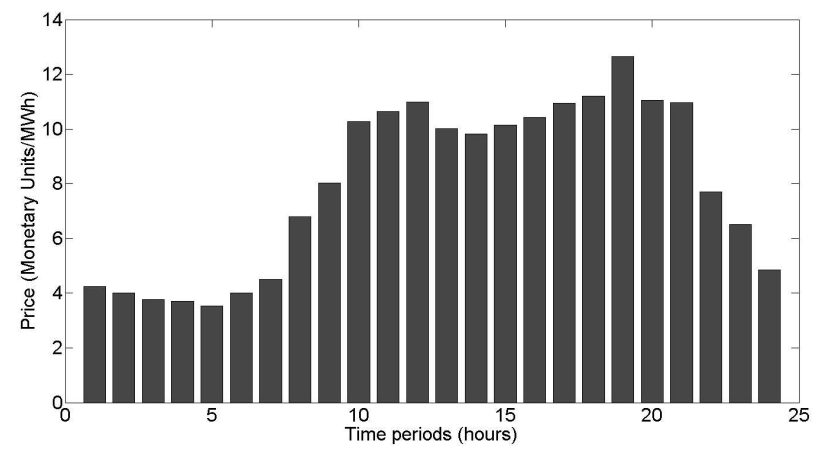

Fig. 4. Hourly nodal price for bus 1

As an example, the hourly generation level for three representative generators is shown in Fig. 5. The production is higher at the end of the day when the demand is increased, starting-up the most expensive generators. On the other hand, the most inexpensive generators are running the whole day.

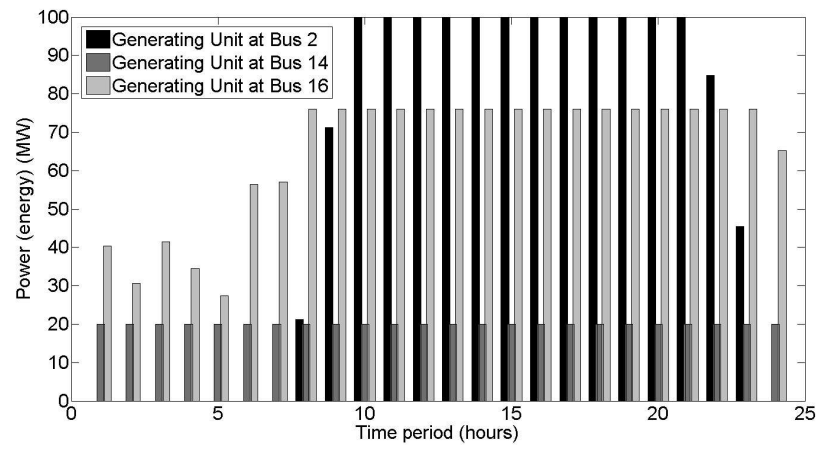

Fig. 5. Hourly generation level

Some results for bilateral contracts are given in Table II. For contract 2, the supplier buys energy from the market in the early time periods due to the market price being lower than the limit established in the contract. However, for both contracts the suppliers sell energy to the market in the intermediate time periods due to the market price being higher than the limit established in the contract.

Finally, the total EVs charging and discharging is shown in Fig. 6. The EVs aggregator takes advantage of low purchase prices, for EVs charging, biding high in the early time periods 
TABLE II

BILATERAL CONTRACTS RESULTS

\begin{tabular}{ccccc}
\hline \multicolumn{5}{c}{ Bilateral contract 1 } \\
\hline Period & Market price & $\varepsilon_{t}^{b}$ & $\vartheta_{t}^{b}$ & Agreement \\
\hline \hline 4 & 3.68 & 4 & 8 & Buy 2.0 MWh \\
\hline 14 & 9.79 & 4 & 8 & Sell 3.0 MWh \\
\hline \multicolumn{5}{c}{ Bilateral contract 2} \\
\hline 19 & 12.57 & 3 & 9 & Sell 4.5 MWh \\
\hline
\end{tabular}

and at the end of the day. However, the EVs aggregator bids high at demand peaks to get additional benefits.

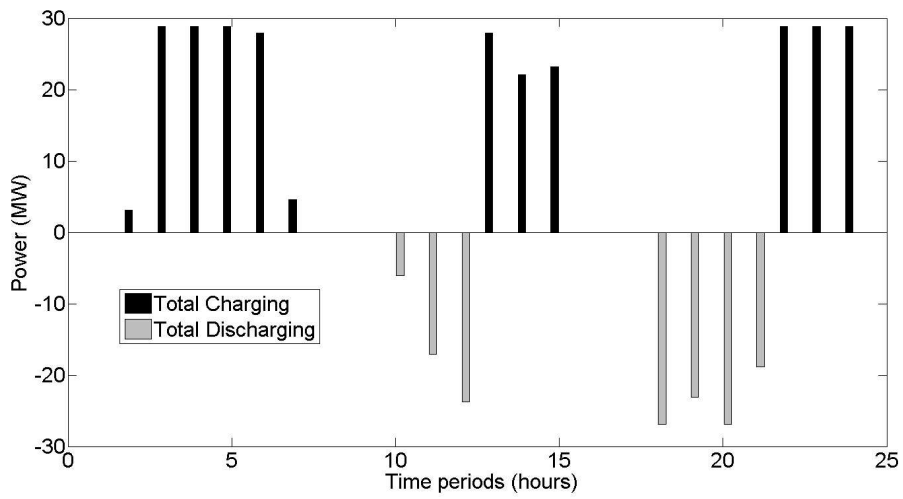

Fig. 6. EVs total charging/discharging

The EVs aggregator's offer mechanism allows for benefits for the grid so the EVs charging is used to fill valleys meanwhile the EVs discharging is used for peak shaving. Fig. 7 shows the demand curves for two different penetration levels.
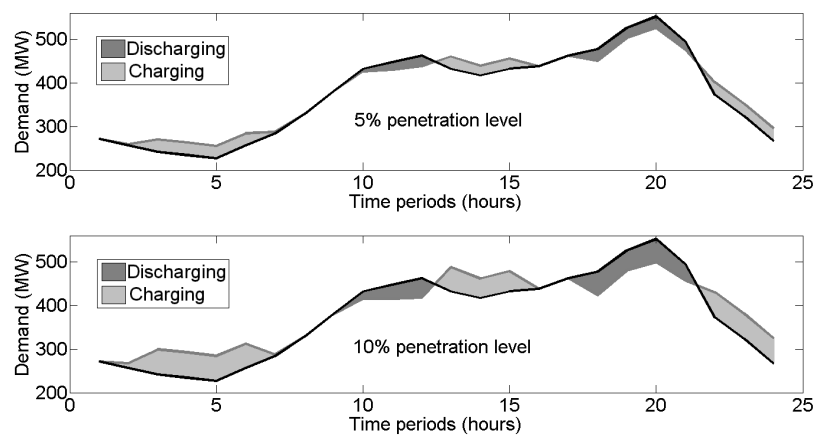

Fig. 7. Demand curves with EVs for two penetration levels

The economical benefits can be assessed through an average price $\pi_{m}$ defined as:

$$
\pi_{m}=\sum_{t=1}^{24} \sum_{n=1}^{24} \frac{\left(\pi_{t, n}^{b} \cdot E_{t, n}^{b}-\pi_{t, n}^{s} \cdot E_{t, n}^{s}\right)}{\left(E_{t, n}^{b}-E_{t, n}^{s}\right)}
$$

where $\pi_{t, n}^{b}$ and $\pi_{t, n}^{s}$ are the buying and selling nodal prices and $E_{t, n}^{b}$ and $E_{t, n}^{s}$ the corresponding amounts of energy, respectively.
For the presented case study a value of 1.01 m.u./MWh was obtained for $\pi_{m}$; this value can be compared with a different simulation for which V2G was not permitted and the resulting $\pi_{m}$ was 4.19 .

\section{CONCLUSION}

An optimization-based model for market-clearing, under a single operator management, has been presented. It incorporates the common participants in energy markets along with an upcoming entity in smart grids, the EVs aggregator.

It has been shown that depending on EVs aggregator's offer mechanism both the aggregator and the system can get benefits. The EVs aggregator can get lower prices for EVs charging and higher income through $\mathrm{V} 2 \mathrm{G}$, also satisfying the mobility requirements. A better final demand curve can be obtained as well, allowing the system to work more efficiently.

The effect of EVs pattern uncertainties and congestion in the market are proposed as future work.

\section{ACKNOWLEDGEMENT}

The authors would like to acknowledge the financial support from the Ministerio de Economía y Competitividad through project ENE-2011-27495.

\section{REFERENCES}

[1] R. Bessa and M. Matos, "Economic and technical management of an aggregation agent for electric vehicles: a literature survey," European Trans. Electrical Power, vol. 22, no. 3, 2012.

[2] T. G. S. Román, I. Momber, M. R. Abbad, and Álvaro Sánchez Miralles, "Regulatory framework and business models for charging plug-in electric vehicles: Infrastructure, agents, and commercial relationships," Energy Policy, vol. 39, no. 10, pp. 6360 - 6375, 2011.

[3] D. Wu, D. Aliprantis, and L. Ying, "Load scheduling and dispatch for aggregators of plug-in electric vehicles," IEEE Trans. Smart Grid, vol. 3, no. 1, pp. 368-376, 2012.

[4] R. Bessa and M. Matos, "Global against divided optimization for the participation of an EV aggregator in the day-ahead electricity market. part I: Theory," Electr. Power Syst. Res., vol. 95, no. 0, pp. 309 - 318, 2013.

[5] — - "Global against divided optimization for the participation of an EV aggregator in the day-ahead electricity market. part II: Numerical analysis," Electr. Power Syst. Res., vol. 95, no. 0, pp. 319 - 329, 2013.

[6] T. K. Kristoffersen, K. Capion, and P. Meibom, "Optimal charging of electric drive vehicles in a market environment," Applied Energy, vol. 88, no. 5, pp. 1940 - 1948, 2011.

[7] N. Rotering and M. Ilic, "Optimal charge control of plug-in hybrid electric vehicles in deregulated electricity markets," IEEE Trans. Power Syst., vol. 26, no. 3, pp. 1021-1029, 2011.

[8] O. Sundström and C. Binding, "Planning electric-drive vehicle charging under constrained grid conditions and perspectives," in Proc. 2010 Power System Technology Conference.

[9] P. Balram, T. Le Anh, and L. Bertling Tjernberg, "Effects of plug-in electric vehicle charge scheduling on the day-ahead electricity market price," in Proc. 2012 Innovative Smart Grid Technologies Europe.

[10] M. Ortega-Vazquez, F. Bouffard, and V. Silva, "Electric vehicle aggregator/system operator coordination for charging scheduling and services procurement," IEEE Trans. on Power Syst.,, vol. 28, no. 2, pp. 1806$1815,2013$.

[11] E. Sortomme and M. El-Sharkawi, "Optimal charging strategies for unidirectional vehicle-to-grid," IEEE Trans. Smart Grid, vol. 2, no. 1, pp. 131-138, 2011.

[12] M. Madrigal and V. Quintana, "A security-constrained energy and spinning reserve markets clearing system using an interior-point method," IEEE Trans. Power Syst., vol. 15, no. 4, pp. 1410-1416, 2000.

[13] P. Subcommittee, "IEEE reliability test system," IEEE Trans. on Power App. Syst.,, vol. PAS-98, no. 6, pp. 2047-2054, 1979. 